\title{
OFFICERS AND LECTURERS OF THE SOCIETY
}

\section{PRESIDENTS}

J. H. Van Amringe, 1889-1890

J. E. McClintock, 1891-1894

G. W. Hill, 1895-1896

Simon Newcomb, 1897-1898

R. S. Woodward, 1899-1900

E. H. Moore, 1901-1902

T. S. Fiske, 1903-1904

W. F. Osgood, 1905-1906

H. S. White, 1907-1908

Maxime Bôcher, 1909-1910

H. B. Fine, 1911-1912

E. B. Van Vleck, 1913-1914

E. W. Brown, 1915-1916

L. E. Dickson, 1917-1918

Frank Morley, 1919-1920

G. A. Bliss, 1921-1922

Oswald Veblen, 1923-1924

G. D. Birkhoff, 1925-1926

Virgil Snyder, 1927-1928

E. R. Hedrick, 1929-1930

L. P. Eisenhart, 1931-1932

\author{
A. B. Coble, 1933-1934 \\ Solomon Lefschetz, 1935-1936 \\ R. L. Moore, 1937-1938 \\ G. C. Evans, 1939-1940 \\ Marston Morse, 1941-1942 \\ M. H. Stone, 1943-1944 \\ T. H. Hildebrandt, 1945-1946 \\ Einar Hille, 1947-1948 \\ J. L. Walsh, 1949-1950 \\ John von Neumann, 1951-1952 \\ G. T. Whyburn, 1953-1954 \\ R. L. Wilder, 1955-1956 \\ Richard Brauer, 1957-1958 \\ E. J. McShane, 1959-1960 \\ Deane Montgomery, 1961-1962 \\ J. L. Doob, 1963-1964 \\ A. A. Albert, 1965-1966 \\ C. B. Morrey, Jr., 1967-1968 \\ Oscar Zariski, 1969-1970 \\ Nathan Jacobson, 1971-1972 \\ Saunders Mac Lane, 1973-1974
}

\section{SECRETARIES}

T. S. Fiske, 1888-1895

F. N. Cole, 1896-1920

R. G. D. Richardson, 1921-1940

J. R. Kline, 1941-1950

\section{TREASURERS}

T. S. Fiske, 1890-1891

Harold Jacoby, 1892-1894

R. S. Woodward, 1895-1896

Harold Jacoby, 1897-1899

W. S. Dennett, 1900-1907

J. H. Tanner, 1908-1920

W. B. Fite, 1921-1929
G. W. Mullins, 1930-1936

P. A. Smith, 1937

B. P. Gill, 1938-1948

A. E. Meder, Jr. 1949-1964

W. T. Martin, 1965-1973

F. P. Peterson, 1973- 


\section{GIBBS LECTURERS}

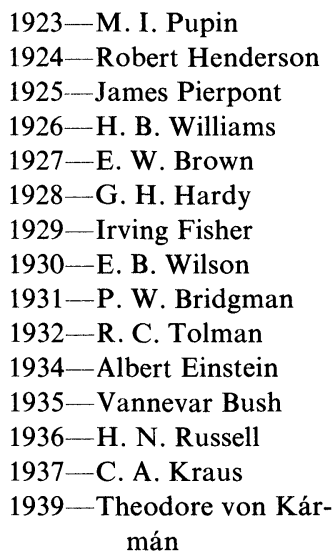

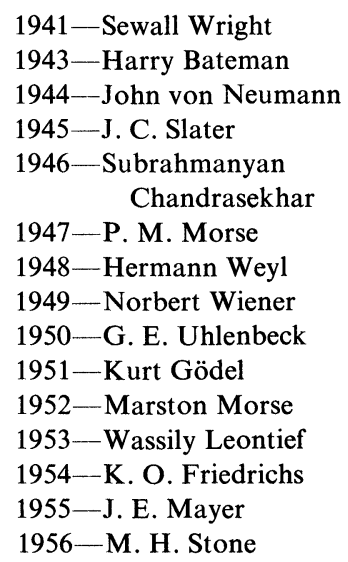

\author{
1958 - H. J. Muller \\ 1959-J. M. Burgers \\ 1960-Julian Schwinger \\ 1961--J. J. Stoker \\ 1962 -C. N. Yang \\ 1963 - C. E. Shannon \\ 1964-Lars Onsager \\ 1965 - D. H. Lehmer \\ 1966-Martin Schwarzschild \\ 1967-Mark Kac \\ 1968-E. P. Wigner \\ 1969 - R. L. Wilder \\ 1970 -W. H. Munk \\ 1971-E. F. F. Hopf \\ 1972 -F. J. Dyson \\ 1973 - J. K. Moser
}

\section{COLLOQUIUM LECTURERS}

\begin{tabular}{|c|c|}
\hline 1896-James Pierpont & 1928 -A. B. Coble \\
\hline Maxime Bôcher & 1929-R. L. Moore \\
\hline 1898--W. F. Osgood & 1930-Solomon Lefschetz \\
\hline A. G. Webster & 1931 - Marston Morse \\
\hline 1901-Oskar Bolza & $1932-J$. F. Ritt \\
\hline E. W. Brown & 1934-R. E. A. C. Paley \\
\hline 1903-H. S. White & Norbert Wiener \\
\hline F. S. Woods & 1935-H. S. Vandiver \\
\hline E. B. Van Vleck & 1936-E. W. Chittenden \\
\hline 1906--E. H. Moore & 1937-John von Neumann \\
\hline E. J. Wilczynski & 1939-A. A. Albert \\
\hline Max Mason & M. H. Stone \\
\hline 1909-G. A. Bliss & 1940-G. T. Whyburn \\
\hline Edward Kasner & 1941-Oystein Ore \\
\hline 1913-L. E. Dickson & 1942 - R. L. Wilder \\
\hline W. F. Osgood & 1943--E. J. McShane \\
\hline 1916-G. C. Evans & 1944-Einar Hille \\
\hline Oswald Veblen & 1945-Tibor Radó \\
\hline 1920 - G. D. Birkhoff & 1946-Hassler Whitney \\
\hline F. R. Moulton & 1947-Oscar Zariski \\
\hline 1925-L. P. Eisenhart & 1948-Richard Brauer \\
\hline Dunham Jackson & 1949--G. A. Hedlund \\
\hline 1927--E. T. Bell & 1951 -Deane Montgomery \\
\hline Anna Pell-Wheeler & 1952-Alfred Tarski \\
\hline
\end{tabular}

\author{
1953-Antoni Zygmund \\ 1955 - Nathan Jacobson \\ 1956-Solomon Bochner \\ 1957-N. E. Steenrod \\ 1959-J. L. Doob \\ 1960-S. S. Chern \\ 1961 - G. W. Mackey \\ 1963 - Saunders Mac Lane \\ 1964 - C. B. Morrey, Jr. \\ 1965-A. P. Calderón \\ 1967 - Samuel Eilenberg \\ 1968-D. C. Spencer \\ J. W. Milnor \\ $1969-$ R. H. Bott \\ Harish-Chandra \\ 1970-R. H. Bing \\ 1971-Lipman Bers \\ Armand Borel \\ 1972 - Stephen Smale \\ John T. Tate \\ 1973-M. F. Atiyah \\ E. A. Bishop \\ F. E. Browder
}

